\title{
Significance of tumour markers in cancer of gall bladder
}

\author{
Sharada R. Kankonkar ${ }^{1^{*}}$, S. V. Joshi ${ }^{2}$, R. R. Deshpande ${ }^{2}$ \\ ${ }^{1}$ Tissue Typing Laboratory, Medical Research Centre, Bombay Hospital Trust, Mumbai, India; \\ *Corresponding author: kankonkar@yahoo.com. \\ ${ }^{2}$ Medical Research Centre, Bombay Hospital Trust, Mumbai, India
}

Received 24 October 2012; revised 28 November 2012; accepted 11 December 2012

\begin{abstract}
In India, GI tract cancer is one of the ten leading cancers. Among Indian males it stands second to oral cancer and in females, it shares the third place. Most common malignant disorder of GIT is seen in our country that of liver, bile, gall bladder, pancreas, bile-duct and colorectal. Aim: To see the significance of tumour markers in gall bladder cancer. Materials and Methods: This study comprise 225 cases of GI tract cancers was carried for more than two years. Of these, 22 subjects had gallbladder cancer. Tumour markers viz. CA19-9, CEA and AFP were assayed pre and post-operative cases and their role in gallbladder cancer was evaluated. Results: It was observed that serum concentration of CA 19-9 increased with advancing stage, but the same is not true for AFP and CEA. Sensitivity of these markers AFP, CA 19-9 and CEA in the detection of gall bladder cancer was determined. CA 19-9 is the most sensitive of all the three tumour markers in the detection of gall bladder cancer. Conclusion: The combination of CA19-9 and CT (or US) is a reasonable, cost-effective, noninvasive approach to establishing the diagnosis of pancreatic, cholangitic, or biliary cancer in nonicteric patients.
\end{abstract}

Keywords: CA19-9; CEA; AFP; Gall Bladder Cancer

\section{INTRODUCTION}

Today, cancer is a vast medical problem. In mortality, it stands second only to cardiovascular diseases. The cancer incidences rise steeply with age so that over the age of 60 years, 3 in every 100 men develop the disease.

In India, GI tract cancer is one of the ten leading cancers; among males it stands second to oral cancer, while in females it shares the third place along with oral cavity cancer, the first two being cervix and breast cancer.

Most common malignant disorder of GIT is seen in our country that of liver, bile, gall bladder, pancreas, bileduct and colorectal.

Worldwide, prominent incidences, geographic variability and their correlation with prevalence of choleithiasis and Gall Bladder Cancer (GBC) have been reported. High rates of GBC was seen in South American countries, particularly Chile, Bolivia, and Ecuador, as well as some areas of India, Pakistan, Japan and Korea [1,2].

The Gallbladder cancer (GBC) is an uncommon but highly fatal malignancy; around 5000 new cases are diagnosed every year in the United States. The majority of such cases were found, incidentally in patients undergoing exploration for cholelithiasis and a tumor could be found in 1 to 2 percent of such cases [3,4].

Satyanarayanan reported significant increasing trends of gall bladder cancer in females [5].

The poor prognosis of gall bladder cancer could be anatomical position of gall bladder and nonspecific symptoms of disease.

Indian studies have reported, life style, reproductive factors, risk factors and role of sonography in gall bladder cancer [6,7]. Increasing trend of gall bladder cancer was observed among females [8]. However, role of tumour markers was documented in a very few single case studies [9-11]. Khan et al. [12] in their study from Aligarh evaluated 52 cases, their risk factors and prognosis of gallbladder cancer. According to Canil et al. [13] tumour markers have the potential to influence clinical decisions at various stages of cancer treatment. They may be used in screening, prognosis, detection of early relapse and monitoring cancer therapy.

Three Tumour Markers viz. CA 19-9, CEA and AFP were selected for the present study.

CA 19-9 tumour marker is used for measurement of 1116-NS-19-9 reactive determinants in human serum. It is used for diagnosis and measurement of patients with pancreatic carcinoma having sensitivity $70 \%-87 \%$. It has been reported that value above $1000 \mathrm{U} / \mathrm{ml}$. almost always have distal metastasis. The value of CA 19-9 is 
frequently elevated in the serum of subjects with various gastrointestinal malignancies, such as pancreatic, colorectal, gastric and hepatic carcinomas. Increased values have also been observed in non-malignant conditions such as hepatic, cirrhosis, pancreatitis.

CEA tumour marker (Carcinoembryonic Antigen) is used is an aid in the prognosis and measurement of patients' value in whom changing concentrations of CEA are observed. This is a broad specificity tumour marker

Quantitative determination of AFP (Alpha-feto-Protein) is used as an aid in the management of patients with non-seminomatous testicular cancer, neural tube defects and liver cirrhosis.

\section{MATERIAL AND METHODS}

This study was carried for more than two years. (20042006) and was based on a retrospective sample analysis of 225 cases of GI tract cancers. Of these, 22 subjects had gallbladder cancer. As far as Ethic approval is concerned, these cases were referred to our Laboratory by various Clinicians of the Hospital Bombay (indoor/ OPD). The Lab was not directly involved with patients. These cases come for required investigation with referring Doctor's note and clinical history. Hence, Ethical approval is not specified in the present study. These are scientific findings we want to share with. Tumour markers viz. CA19-9, CEA and AFP were assayed pre and post-operatively in serum of patients with carcinoma of the gallbladder, using ELISA(IMX System) technique. Clinical presentation, laboratory investigations, treatment given, operative findings and histopathological findings were reviewed. Microparticle Enzyme Immunoassay (MEIA) method on Abbotts Axsym analyzer was used for this study. $5 \mathrm{ml}$ of patients serum was collected and quantitative determination of each sample was done. Statistical analysis was done in terms of percentages only to correlate the tumour markers and stages of gall bladder cancer.

\section{RESULTS}

Out of 225 patients included in the study, 22 patients suffered from gall bladder cancer [Figure 1].

$$
\mathrm{M}: \mathrm{F}=12: 10=6: 5
$$

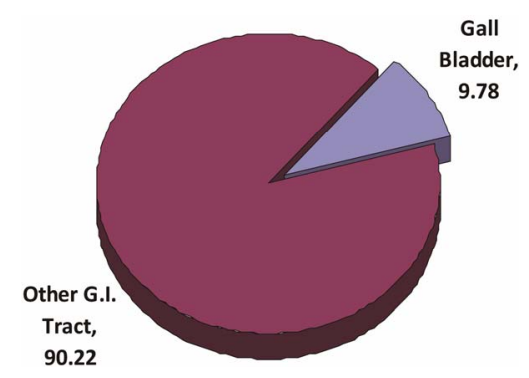

Figure 1. Distribution of patients.
Age range $=38$ to 73 years.

Average age $=53.45 \pm 11.70$ years.

The patients were classified on the basis of TNM staging. Stage I-0, Stage II-4, Stage III-9, Stage IVA-5, Stage IVB-4 [Table 1].

It can be seen that serum concentration of CA 19-9 increases with advancing stage, but the same is not true for tumour markers AFP and CEA (Table 1 and Figure 2).

Sensitivity of tumour markers AFP, CA 19-9 and CEA in the detection of gall bladder cancer was determined. CA 19-9 was the most sensitive of all the three tumour markers of gall bladder cancer (Figure 2).

A follow-up study was carried out. One gall bladder cancer patient with peritoneal metastasis died. Another patient reported for follow-up, whose serum CA 19-9 concentration was studied.

A follow-up study was carried out. For six patients. Their serum CA 19-9 concentrations were studied (Table 2).

In the present study, there was no patient at stage I of gall bladder cancer. It can be seen that all the 22 patients with gall bladder cancer showed elevated CA 19-9 levels. Elevated levels of AFP were seen in patients who were at stage III and stage IVA of the disease but the elevations were not $100 \%$. In case of CEA the percentage elevations decreased with increasing stage.

\section{DISCUSSION}

Prospective analysis of gastrointestinal cancers was 9.78\% of gall bladder. Among the three tumour markers, CA 19-9 was the most sensitive in the detection of gall bladder cancer.

Results showed both the genders suffered from cancer of gall bladder though literature quoted high incidence of gall bladder cancers in females [14,15]. However, in our series male preponderance was observed. It could be due to the selective sample referred to this laboratory.

Though Carcinoma of the gallbladder is common in tobacco chewers [15]. There was no correlation between tobacco and cancer in our study.

In the present series, CA19-9 was correlated with

Table 1. Percentage of gall bladder cancer patients with elevated tumour marker levels.

\begin{tabular}{cccc}
\hline Stage & AFP & CA 19-9 & CEA \\
\hline I & - & - & - \\
II & $0.00 \%(0 / 1)$ & $100.00 \%(4 / 4)$ & $100.00 \%(2 / 2)$ \\
III & $40.00 \%(2 / 5)$ & $100.00 \%(9 / 9)$ & $77.77 \%(7 / 9)$ \\
IVA & $80.00 \%(4 / 5)$ & $100.00 \%(5 / 5)$ & $60.00 \%(3 / 5)$ \\
IVB & $0.00 \%(0 / 1)$ & $100.00 \%(4 / 4)$ & $50.00 \%(1 / 2)$ \\
\hline
\end{tabular}

Note: Figures in the parentheses state number of patients. 


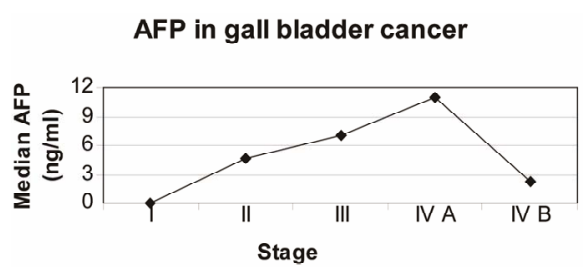

(a)

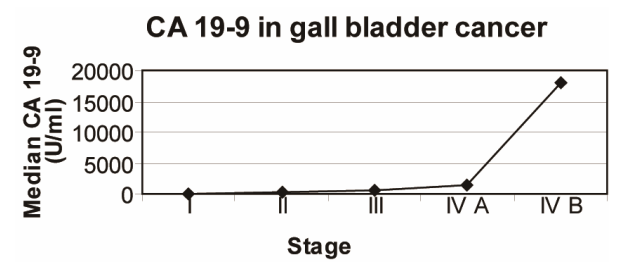

(b)

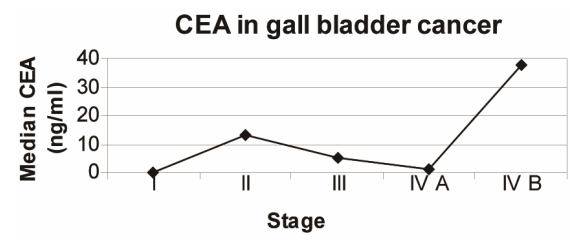

(c)

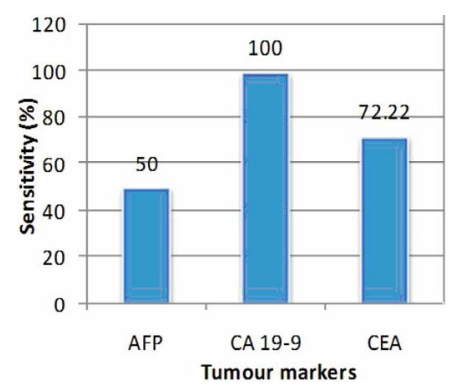

(d)

Figure 2. Sensitivity of tumour markers, correlation between stages of gall bladder cancer and tumour markers.

Table 2. Post treatment CA19-9 in Gall Bladder cancer.

\begin{tabular}{cccc}
\hline Sr. No. & $\begin{array}{c}\text { Pre-treatment } \\
\text { CA19-9values }\end{array}$ & Treatment taken & $\begin{array}{c}\text { Post-treatment } \\
\text { CA 19-9 values }\end{array}$ \\
\hline 1. & 150.10 & Surgery & 11342.00 \\
\hline
\end{tabular}

stages of gall bladder cancer. Similar findings were reported by Shukla et al. (15). However, Vij et al observed that serum levels of CEA and AFP did not have any diagnostic or prognostic significance in the management of Cancer of gall bladder [16].

Most of the documented cases of gall bladder cancer were of single case study.

Ono et al. [10] reported rare 63-yrs-old case with metastases of the liver, based on ultrasonography and computed tomography of the abdomen showing multiple tumorous lesions in the liver Laboratory data showed high levels of tumor markers: $4647.4 \mathrm{ng} / \mathrm{ml}$ AFP, 9987.1 ng/ml CEA, 11704.0 U/ml CA19-9, 847.6 U/ml.

A 73-year-old woman with gallbladder carcinoma infiltrating to the liver presenting high serum values of AFP and CEA was reported. Serum values of AFP and CEA were remarkably high (165,000 ng/ml and 1070 $\mathrm{ng} / \mathrm{ml})$. Immunohistochemically the tumor cells were stained for AFP and CEA by the PAP method [9].

Tatyen et al. reported a 64-year-old man with an elevated CA 19-9 of $5791 \mathrm{U} / \mathrm{ml}$ and radiological findings were suggestive of metastatic gallbladder carcinoma [11]. The patient underwent cholecystectomy and excision of a common bile duct stricture, with hepaticojejunostomy and liver biopsy.

Although no single procedure or combination of procedures was found to detect early, lesions, CA19-9 was clearly a clinically useful adjunct to imaging in nonjaundiced patients suspected of having these malignancies.

We observed correlation between the serum concentration of CA 19-9 and with advancing stage, but the same was not true for tumour markers AFP and CEA.

Shukla 2006 from Varanasi reported 55 cases and 8 healthy controls presenting to a single surgical unit of the University Hospital, Varanasi, CA19-9, CA15-3 and CA 125 were assayed preoperatively in serum of patients with carcinoma of the gallbladder (39), cholelithiasis (16) and healthy controls (8) using ELISA technique. CA19-9 was 211.27 vs 86.06 uml [17].

In the present study, sensitivity of tumour markers AFP, CA 19-9 and CEA in detection of gall bladder cancer was determined. CA 19-9 was found most sensitive of all the three tumour markers.

Another study by Brockman et al showed 76.5\% sensitivity of CA 19-9 for pancreatic carcinomas and a specificity of $96.4 \%$ [18].

The sensitivity and specificity of Ca 19-9 was 0.66 and 0.90 respectively. Carcinoembryonic antigen was over $2.5 \mathrm{ng} / \mathrm{ml}$ in 25 patients with cancer (56\%) and its sensitivity and specificity was 0.75 and 0.71 respectively [19].

The poor prognosis of carcinoma of the gallbladder (CAGB) could be due to delayed presentation and absence of specific clinical findings in the early stages. To ascertain whether the commonly available serum tumour markers (carcino-embryonic antigen-CEA and alpha foeto protein-AFP) could be used for distinguishing carcinoma of gall bladder from others [16].

In our study, one patient showed higher values (11342 $\mathrm{U} / \mathrm{ml}$ ) of CA19-9 after surgery which was suggestive of progression of the disease.

Patients who showed normal value of CA 19-9 postoperatively, live longer than those who do not. Further, serially used tumour marker helped predicting recurrence of disease prior to radiographic or clinical findings. The 
CA 19-9 is currently the "gold" standard marker for pancreatic cancer, against which other assays in this field will be judged [20].

The sensitivity of tumour markers AFP, CA 19-9 and CEA in the detection of gall bladder cancer was determined and was found that CA 19-9 was the most sensitive of all the three tumour markers. It was also seen that serum concentration of CA 19-9 increased with advancing stage of disease, but the same was not true for tumour markers AFP and CEA.

Conclusion: The combination of CA19-9 and CT (or US) is a reasonable, cost-effective, noninvasive approach to establishing the diagnosis of gall bladder cancer.

\section{ACKNOWLEDGEMENTS}

We acknowledge co-operation of staff of Tissue Typing Laboratory.

\section{REFERENCES}

[1] Diehl, A.K. (1980) Epidemiology of gallbladder cancer: A synthesis of recent data. Journal of the National Cancer Institute, 65, 1209-1214.

[2] Strom, B.L, Soloway, R.D, Rios-Dalenz, J.L. and Rodriguez-Martinez, H.A. (1995) Risk factors for gallbladder cancer. An international collaborative case-control study. Cancer, 76, 1747-1756. doi:10.1002/1097-0142(19951115)76:10<1747::AID-CN CR2820761011>3.0.CO;2-L

[3] Yamaguchi, K., Chijiiwa, K., Ichimiya, H. and Sada, M. (1996) Gallbladder carcinoma in the era of laparoscopic cholecystectomy. Archives of Surgery, 131, 981-984. doi:10.1001/archsurg.1996.01430210079015

[4] Marinus, J.P.G., van Kroonenburgh, H. and Pasmans L.M. (1991) A prospective analysis of 1518 laparoscopic cholecystectomies. The southern surgeons club. The New England Journal of Medicine, 324, 1073-1078. doi:10.1056/NEJM199104183241601

[5] Satyanarayana, L. and Asthana, S. (2008) Life time risk for development of ten major cancers in India and its trends over the years 1982 to 2000. Indian Journal of Medical Sciences, 62, 35-44. doi:10.4103/0019-5359.39365

[6] Pandey, M. and Shukla, V.K. (2003) Lifestyle, parity, menstrual and reproductive factors and risk of gallbladder cancer. European Journal of Cancer Prevention, 12, 269272. doi:10.1097/00008469-200308000-00005

[7] Pandey, M., Sood, B.P., Shukla, R.C. and Aryya, N.C. (2000) Carcinoma of the gallbladder: Role of sonography in diagnosis and staging. Journal of Clinical Ultrasound, 28, 227-232. doi:10.1002/(SICI)1097-0096(200006)28:5<227::AID-JC $\underline{\mathrm{U} 4>3.0 . \mathrm{CO} ; 2-4}$
[8] Kumar, A. and Aggarwal, S. (1994) Carcinoma of the gallbladder: CT findings in 50 cases. Abdominal Imaging, 19, 304-308. doi:10.1007/BF00198184

[9] Sugaya, Y., Sugaya, H., Kuronuma, Y., Hisauchi, T. and Harada, T. (1989) A case of gallbladder carcinoma producing both alpha-fetoprotein (AFP) and carcinoembryonic antigen (CEA). Gastroenterologia Japonica, 24, 325331.

[10] Ono, T., Komatsu, M., Hoshino, T., Ishii, T., Fujii, T., Oshima, S., et al. (1996) Alpha-fetoprotein, carcinoembryonic antigen, and carbohydrate antigen 19-9-producing gallbladder cancer. Journal of Gastroenterology, 31, 742-746. doi:10.1007/BF02347628

[11] Clarke, T., Matsuoka, L., Jabbour, N., Mateo, R., Genyk, Y., Selby, R., et al. (2007) Gallbladder mass with a carbohydrate antigen 19-9 level in the thousands: Malignant or benign pathology? Report of a Case Surgery Today, 37940, 342-344. doi:10.1007/s00595-006-3377-4

[12] Khan, R.A., Wahab, S., Khan, M.A., Siddiqui, S. and Maheshwari, V. (2010) Advanced presentation of gallbladder cancer: Epidemioclinico pathological study to evaluate the risk factors and assess the outcome. Journal of the Pakistan Medical Association, 60, 217-219.

[13] Canil, C.M. and Tannock, I.F. (2002) Doctor's dilemma: Incorporating tumor markers into clinical decision-making. Seminars in Oncology, 29, 286-93. doi:10.1053/sonc.2002.32904

[14] Sharma, R.G., Kumar, R., Jain, S., Jhajhria, S., Gupta, N., Gupta, S.K., et al. (2009) Distribution of malignant neoplasms reported at different pathology centers and hospitals in Jaipur, Rajasthan. Indian Journal of Cancer, 46, 323-330. doi:10.4103/0019-509X.55553

[15] Shukla, V.K., Chauhan, V.S., Mishra, R.N. and Basu, S. (2008) Lifestyle, reproductive factors and risk of gallbladder cancer. Singapore Medical Journal, 49, 912-915.

[16] Vij, U. and Baskaran, V. (2001) Value of serum CEA and AFP in the diagnosis and prognosis of carcinoma gallbladder. Tropical Gastroenterology, 22, 227-229.

[17] Shukla, V.K., Gurubachan, Sharma, D., Dixit, V.K. and Usha (2006) Diagnostic value of serum CA242, CA 19-9, CA $15-3$ and CA 125 in patients with carcinoma of the gallbladder. Tropical Gastroenterology, 27, 160-165.

[18] Brockmann, J., Emparan, C., Hernandez, C.A., Sulkowski, U., Dietl, K.H., Menzel, J., et al. (2000) Gallbladder bile tumor marker quantification for detection of pancreatobiliary malignancies. Anticancer Research, 20, 49414947.

[19] De Aretxabala, X., Riedeman, J.P., Roa, I., Wenzel, C., Inostroza, J., Burgos, L., et al. (1996) CA 19-9 and carcinoembryonic antigen in gallbladder cancer. Revista Médica de Chile, 124, 11-20.

[20] Steinberg, W. (1990) The clinical utility of the CA 19-9 tumor-associated antigen. The American Journal of Gastroenterology, 85, 350-355. 